\title{
Synthesis of New Pyrazole Derivatives Containing Quinoline Moiety via Chalcones: A Novel Class of Potential Antibacterial and Antifungal Agents
}

\author{
G. MANJUNATH, M. MAHESH, G. BHEEMARAJU and P. VENKATA RAMANA* \\ Department of Chemistry, Sri Krishnadevaraya University, \\ Ananthapuramu-515003, Andhra Pradesh, India \\ ramanapv54@gmail.com
}

Received 29 September 2015 / Accepted 28 October 2015

\begin{abstract}
In the present investigation a new class of quinoline derivatives containing pyrazole moiety have been designed and synthesized by condensation of 2-(quinolin-8-yloxy)acetohydrazide with various chalcones. These newly synthesized quinoline derivatives containing pyrazole moiety were screened for their minimum inhibitory concentration by antibacterial activity against two kinds of strains i.e. Staphylococcus aureus, Escherichia coli and antifungal activity against Aspergillus niger. The results showed that some of the compounds exhibited moderate to good antibacterial activity against both the strains and a few compounds were active in antifungal activity. The structure-activity relationships were briefly discussed. The studies indicated that variation of substituent in the aromatic rings changes the antibacterial activity and compounds containing electron withdrawing groups exhibited potential antifungal activity.
\end{abstract}

Keywords: Quinolin-8-ol, Ethyl chloroacetate, Hydrazine hydrate, Chalcone, Pyrazole, Antibacterial activity and Antifungal activity

\section{Introduction}

A wide range of antimicrobial agents have been discovered to prolong the lifespan of people but unfortunately microbial resistance resulted in a dwindling pool of effective antibiotics. At this instance, resistance to existing antifungal agents is also a major threat and hence there is a pressing need for the development of new antimicrobial agents which may be effective against the resistant microbes.

Quinoline $^{1-5}$ has its own prominence in drug discovery programs. Quinoline along with its derivatives is reported to exhibit a wide spectrum of biological properties such as

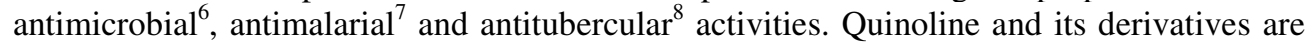
widely used as fungicides, biocides, antibiotics, alkaloids, dyes, rubber chemicals and flavoring agents. They are also used in manufacturing oil soluble dyes, food colorants, pharmaceuticals, $\mathrm{pH}$ indicators and other organic compounds. 
Pyrazoles $^{9-13}$ are potential bioactive agents due to their wide spectrum of pharmacological activities like anti-inflammatory, antimicrobial, antihypertensive, antitumor, anticonvulsant, antitubercular, hypoglycemic and analgesic. Chalcones ${ }^{14-18}$ represents important building blocks for both natural and synthetic bioactive compounds. Chalcones are fine synthons for different heterocyclic rings. So it was planned to synthesize some new pyrazoles from chalcones with the hope that they may possess better antimicrobial activities.

Looking at the importance of quinoline and pyrazole nucleus, it was thought that it would be worthwhile to design and synthesize some new quinoline derivatives bearing pyrazole moiety and screen them for potential biological activities.

\section{Experimental}

All chemicals and reagents were procured from Merck India limited. Melting points were determined in open capillary on a Mel-Temp apparatus and are uncorrected. The progress of the reaction was monitored by TLC (silica gel H, BDH, ethyl acetate-hexane, 3:5). The IR spectra were recorded on IR $200 \mathrm{FT}-\mathrm{IR}$ spectrometer as $\mathrm{KBr}$ pellets. The wave numbers were given in $\mathrm{cm}^{-1}$. The ${ }^{1} \mathrm{H}$ NMR spectra were recorded in $\mathrm{CDCl}_{3} / \mathrm{DMSO}-\mathrm{d}_{6}$ on a Jeol JNM $\lambda-400 \mathrm{MHz}$ machine. The ${ }^{13} \mathrm{C}$ NMR spectra were recorded in $\mathrm{CDCl}_{3} / \mathrm{DMSO}-\mathrm{d}_{6}$ on a Jeol JNM spectrometer operating at $125 \mathrm{MHz}$. All chemical shifts were measured in $\delta$ (ppm) using TMS as an internal standard. The mass spectra were recorded on VG $7070 \mathrm{H}$ mass spectrometer. The microanalyses were performed on Perkin-Elmer $240 \mathrm{C}$ elemental analyzer. The microwave syntheses were carried out in a domestic microwave oven (LG MH2548QPS) wherever it was specified.

\section{Biological activity}

The minimum inhibitory concentration ${ }^{19,20}$ of the compounds 7a-o was determined by broth dilution method. The respective clinical strain was spread separately on the Mueller-Hinton broth $^{21}$ medium for antibacterial activity and Sabouraud dextrose agar (SDA) broth for antifungal activity. The synthesized compounds were dissolved in DMSO at different concentrations such as $100,87.5,75,62.5,50,37.5,25,12.5,6.25,3.13,1.56,0.78,0.39$ and $0.19 \mu \mathrm{g} / \mathrm{mL}$ and $2 \mathrm{~mL}$ of these solutions were taken in test tubes. Then $2 \mu \mathrm{L}$ of test organism suspension was added and incubated at $37^{\circ} \mathrm{C}$ for $24 \mathrm{~h}$ for bacteria and $48 \mathrm{~h}$ for fungi studies. The drugs ofloxacin and fluconazole were used as standards for comparison of antibacterial and antifungal activities respectively. The minimum inhibitory concentration was the lowest concentration of test compound that inhibit the visible growth of the organism and was determined in triplicates. The results are tabulated in the Table 2.

\section{Synthesis of ethyl 2-(quinolin-8-yloxy)acetate 2}

A mixture of quinolin-8-ol $1(0.01 \mathrm{~mol})$, ethyl chloroacetate $(0.01 \mathrm{~mol})$, anhydrous $\mathrm{K}_{2} \mathrm{CO}_{3}$ $(1.38 \mathrm{~g}, 0.01 \mathrm{~mol})$ and DMF was stirred at room temperature for $8 \mathrm{~h}$. The reaction mixture was diluted with ice-cold water. The separated solid was filtered, washed with water and recrystallized from ethanol to afford ethyl 2-(quinolin-8-yloxy)acetate 2.

\section{Characterization data of 2}

Yield 80\%. m.p. 56-58 ${ }^{\circ} \mathrm{C}$. IR (KBr) $v_{\max }$ : 3065 (C-H stretch in aromatics), 2920 (C-H stretch in $\left.\mathrm{CH}_{3} / \mathrm{CH}_{2}\right), 1730\left(\mathrm{C}=\mathrm{O}\right.$ stretch), $1215,1015\left(\mathrm{sp}^{2} / \mathrm{sp}^{3} \mathrm{C}-\mathrm{O}\right.$ stretch $) \mathrm{cm}^{-1} .{ }^{1} \mathrm{H}$ NMR (DMSO-d $\left.\mathrm{d}_{6}\right)$ : $\delta 1.28\left(\mathrm{t}, \mathrm{J}=6.8 \mathrm{~Hz}, 3 \mathrm{H}\right.$, ester $\left.\mathrm{CH}_{3}\right), 4.29\left(\mathrm{q}, \mathrm{J}=7.2 \mathrm{~Hz}, 2 \mathrm{H}\right.$, ester $\left.\mathrm{CH}_{2}\right), 4.95\left(\mathrm{~s}, 2 \mathrm{H}, \mathrm{OCH}_{2}\right)$, $6.98\left(\mathrm{~d}, \mathrm{~J}=2.4 \mathrm{~Hz}, 1 \mathrm{H}\right.$, quinoline- $\left.\mathrm{H}_{7}\right), 7.44-7.47\left(\mathrm{~m}, 3 \mathrm{H}\right.$, quinoline $\left.-\mathrm{H}_{3},-\mathrm{H}_{5},-\mathrm{H}_{6}\right), 8.15(\mathrm{dd}, \mathrm{J}=$ 2.0, 6.8, 1.6 Hz, $1 \mathrm{H}$, quinoline- $\left.\mathrm{H}_{4}\right), 8.96\left(\mathrm{dd}, \mathrm{J}=1.6,2.4,2.0 \mathrm{~Hz}, 1 \mathrm{H}\right.$, quinoline- $\left.\mathrm{H}_{2}\right) \mathrm{ppm}$. 
${ }^{13} \mathrm{C}$ NMR (DMSO-d $\left.\mathrm{d}_{6}\right): \delta 13.8\left(\right.$ ester $\left.\mathrm{CH}_{3}\right), 63.2\left(\right.$ ester $\left.\mathrm{CH}_{2}\right), 67.1\left(\mathrm{OCH}_{2}\right), 112.3,121.4$, $122.5,124.0,129.1,136.2,139.2,155.2,153.5,165.6$ (aromatic carbons) ppm. MS m/z: found $231\left[\mathrm{M}^{+}\right]$; calcd. 231. Anal. $\mathrm{C}_{13} \mathrm{H}_{13} \mathrm{NO}_{3}$. Found $\mathrm{C} 66.48$ (67.52), H 5.53 (5.67), N 5.98 (6.06).

\section{Synthesis of 2-(quinolin-8-yloxy)acetohydrazide 3}

A solution of ethyl 2-(quinolin-8-yloxy)acetate 2 ( 0.01 mole) and hydrazine hydrate $(0.015$ mole) in ethanol $(25 \mathrm{~mL})$ was refluxed for $5 \mathrm{~h}$. The excess of solvent was distilled off and the reaction mixture was cooled. The separated solid was filtered, washed with petroleum ether and recrystallized from water to afford $\mathbf{3}$.

\section{Characterization data of $\mathbf{3}$}

Yield 76\%. m.p. 124-126 ${ }^{\circ} \mathrm{C}$. IR $(\mathrm{KBr}) \mathrm{v}_{\max }$ : $3325(\mathrm{~N}-\mathrm{H}$ stretch), 3060 (C-H stretch in aromatics), 2895 (C-H stretch in $\left.\mathrm{CH}_{3} / \mathrm{CH}_{2}\right), 1660\left(\mathrm{C}=\mathrm{O}\right.$ stretch), $1285,1035\left(\mathrm{sp}^{2} / \mathrm{sp}^{3} \mathrm{C}-\mathrm{O}\right.$ stretch) $\mathrm{cm}^{-1} .{ }^{1} \mathrm{H}$ NMR $\left(\right.$ DMSO-d $\left._{6}\right): \delta 3.98\left(\mathrm{~s}, 2 \mathrm{H}, \mathrm{NH}_{2}\right), 4.87\left(\mathrm{~s}, 2 \mathrm{H}, \mathrm{CH}_{2} \mathrm{O}\right), 7.17(\mathrm{dd}, \mathrm{J}=$ 1.2, 5.6, $1.6 \mathrm{~Hz}, 1 \mathrm{H}$, quinoline- $\left.\mathrm{H}_{7}\right), 7.47-7.54\left(\mathrm{~m}, 3 \mathrm{H}\right.$, quinoline- $\left.\mathrm{H}_{3},-\mathrm{H}_{5},-\mathrm{H}_{6}\right), 8.20(\mathrm{dd}, \mathrm{J}=$ 2.0, 6.8, $1.2 \mathrm{~Hz}, 1 \mathrm{H}$, quinoline- $\left.\mathrm{H}_{4}\right), 8.93\left(\mathrm{dd}, \mathrm{J}=1.2,3.2,1.2 \mathrm{~Hz}, 1 \mathrm{H}\right.$, quinoline- $\left.\mathrm{H}_{2}\right), 9.75$ (s, 1H, NH) ppm.

${ }^{13} \mathrm{C}$ NMR (DMSO-d $\left.\mathrm{d}_{6}\right): \delta 67.5\left(\mathrm{OCH}_{2}\right), 112.4,121.6,122.5,123.6,130.0,135.9,139.4$, 153.5, 154.6, 162.7 (aromatic carbons) ppm. MS $\mathrm{m} / z$ : found $217\left[\mathrm{M}^{+}\right]$; calcd. 217. Anal. $\mathrm{C}_{11} \mathrm{H}_{11} \mathrm{~N}_{3} \mathrm{O}_{2}$. Found C 60.14 (60.82), H 4.98 (5.10), N 19.17 (19.34).

General procedure for the synthesis of 1,3-bis(substituted phenyl)prop-2-en-1ones 6a-o

A number of known and novel chalcone derivatives were prepared by Claisen- Schmidt condensation of appropriate aldehyde and acetophenone derivatives.

To a solution of substituted acetophenone $\mathbf{5}(0.01 \mathrm{~mol})$ in ethanol $(20 \mathrm{~mL})$, substituted aromatic benzaldehyde $4(0.01 \mathrm{~mol})$ was added and cooled to $5-10{ }^{\circ} \mathrm{C}$ in an ice bath. To this cold solution, sodium hydroxide (30\%) was added and stirred magnetically for $1 \mathrm{~h}$ and then left overnight or longer, monitored by TLC. The resultant solution was diluted with ice water and acidified with dilute $\mathrm{HCl}$. The chalcone separated as solid was collected by filtration after washing with water and recrystallized from ethanol.

The physical and analytical characterization data of 1,3-bis(substituted phenyl)prop-2en-1-ones 6a-o are presented in Table 1.

\section{Characterization data of $\mathbf{6 a}$}

Recrystallised from ethanol as yellow solid in $91 \%$ yield. m.p. $56-58^{\circ} \mathrm{C}$. IR $(\mathrm{KBr}) v_{\max }: 3064$ (C-H stretch in aromatics), $2938\left(\mathrm{C}-\mathrm{H}\right.$ stretch in $\left.\mathrm{CH}_{3} / \mathrm{CH}_{2}\right), 1675(\mathrm{C}=\mathrm{O}$ stretch $), 1618(\mathrm{C}=\mathrm{C}$ stretch in olefins), $1596(\mathrm{C}=\mathrm{C}$ stretch $) \mathrm{cm}^{-1}$.

${ }^{1} \mathrm{H}$ NMR (DMSO-d $\left.\mathrm{d}_{6}\right): \delta 7.38-7.40(\mathrm{~m}, 3 \mathrm{H}, \mathrm{Ar}-\mathrm{H}), 7.54(\mathrm{~d}, \mathrm{~J}=15.6 \mathrm{~Hz}, 1 \mathrm{H},=\mathrm{CH}-$ $\mathrm{C}=\mathrm{O}), 7.63-7.65(\mathrm{~m}, 2 \mathrm{H}, \mathrm{Ar}-\mathrm{H}), 7.66-7.68\left(\mathrm{~m}, 2 \mathrm{H}, \mathrm{Ar}{ }^{\prime}-\mathrm{H}\right), 7.76-7.78\left(\mathrm{~m}, 1 \mathrm{H}, \mathrm{Ar}{ }^{\prime}-\mathrm{H}\right)$, $7.84-7.86\left(\mathrm{~m}, 2 \mathrm{H}, \mathrm{Ar}{ }^{\prime}-\mathrm{H}\right), 8.04(\mathrm{~d}, \mathrm{~J}=15.6 \mathrm{~Hz}, 1 \mathrm{H},=\mathrm{CH}) \mathrm{ppm}$.

${ }^{13} \mathrm{C}$ NMR (DMSO-d $\left.)_{6}\right): \delta 121.5$ (=C-H adjacent to carbonyl), $145.6(=\mathrm{C}-\mathrm{H}), 126.8$, 128.1, 128.6, 128.8, 129.3, 134.4, 135.3, 138.2 (aromatic carbons), $190.8(\mathrm{C}=\mathrm{O}) \mathrm{ppm}$. MS $m / z$ : found $208\left[\mathrm{M}^{+}\right]$; calcd. 208. Anal. $\mathrm{C}_{15} \mathrm{H}_{12} \mathrm{O}$. Found $\mathrm{C} 86.39$ (86.51), H 5.79 (5.81). 
Table 1. Physical and analytical characterization data of 1,3-bis(substituted phenyl)prop-2en-1-ones 6a-o

\begin{tabular}{cccccc}
\hline S. No. & Compound & $\mathrm{R}_{1}$ & $\mathrm{R}_{2}$ & Colour & Melting point, ${ }^{\circ} \mathrm{C}$ \\
\hline 1 & $\mathbf{6}$ & $-\mathrm{H}$ & $-\mathrm{H}$ & Yellow & $56-58$ \\
2 & $\mathbf{6 b}$ & $-\mathrm{H}$ & $p-\mathrm{OH}$ & Lemon yellow & $172-174$ \\
3 & $\mathbf{6 c}$ & $-\mathrm{H}$ & $o-\mathrm{OH}$ & Golden yellow & $68-70$ \\
4 & $\mathbf{6 d}$ & $-\mathrm{H}$ & $p-\mathrm{Cl}$ & Light yellow & $124-126$ \\
5 & $\mathbf{6 e}$ & $-\mathrm{H}$ & $p-\mathrm{NO}_{2}$ & Dark yellow & $92-94$ \\
6 & $\mathbf{6}$ & $p-\mathrm{OCH}_{3}$ & $-\mathrm{H}$ & Lemon yellow & $126-128$ \\
7 & $\mathbf{6 g}$ & $p-\mathrm{OCH}_{3}$ & $p-\mathrm{OH}$ & Golden yellow & $224-226$ \\
8 & $\mathbf{6}$ & $p-\mathrm{OCH}_{3}$ & $o-\mathrm{OH}$ & Golden yellow & $134-136$ \\
9 & $\mathbf{6 i}$ & $p-\mathrm{OCH}_{3}$ & $p-\mathrm{Cl}$ & Light yellow & $170-172$ \\
10 & $\mathbf{6 j}$ & $p-\mathrm{OCH}$ & $p-\mathrm{NO}$ & Dark yellow & $128-130$ \\
11 & $\mathbf{6 k}$ & $o-\mathrm{OH}$ & $-\mathrm{H}$ & Brownish yellow & $100-102$ \\
12 & $\mathbf{6}$ & $o-\mathrm{OH}$ & $p-\mathrm{OH}$ & Yellow & $182-184$ \\
13 & $\mathbf{6 m}$ & $o-\mathrm{OH}$ & $o-\mathrm{OH}$ & Yellow & $150-152$ \\
14 & $\mathbf{6 n}$ & $o-\mathrm{OH}$ & $p-\mathrm{Cl}$ & Lemon yellow & $236-238$ \\
15 & $\mathbf{6 o}$ & $o-\mathrm{OH}$ & $p-\mathrm{NO}_{2}$ & Chocolate brown & $196-198$ \\
\hline
\end{tabular}

Synthesis of 1-(substituted 3,5-diphenyl-4,5-dihydro-1H-pyrazol-1-yl)-2-(quinolin-8yloxy)ethanones 7a-o

Synthesis of 1-(3,5-diphenyl-4,5-dihydro-1H-pyrazol-1-yl)-2-(quinolin-8-yloxy) ethanone $7 a$

\section{Conventional method}

To a solution of $6 \mathbf{a}(0.01 \mathrm{~mol})$ in glacial acetic acid $(25 \mathrm{~mL}, 0.01 \mathrm{~mol})$, 2-(quinolin-8-yloxy) acetohydrazide $3(0.01 \mathrm{~mol})$ was added. The mixture was refluxed for $6-7 \mathrm{~h}$ and left overnight. The reaction mixture was poured onto crushed ice and the solid mass was filtered, washed with ethanol, dried and purified by recrystallization from ethanol to afford $7 \mathbf{a}$.

\section{Microwave method}

To the solution of $6 \mathbf{a}(0.01 \mathrm{~mol})$ in glacial acetic acid $(25 \mathrm{~mL}, 0.01 \mathrm{~mol})$, 2-(quinolin-8yloxy) acetohydrazide $3(0.01 \mathrm{~mol})$ was added. The mixture was irradiated under MW at $80 \%$ power for 10-12 min and the reaction mixture was allowed to attain room temperature and poured on crushed ice. The precipitated solid mass was filtered, washed with ethanol, dried and purified by recrystallization from ethanol to afford 7a (Figure 1). Compounds 7b-7o are prepared on the same lines.

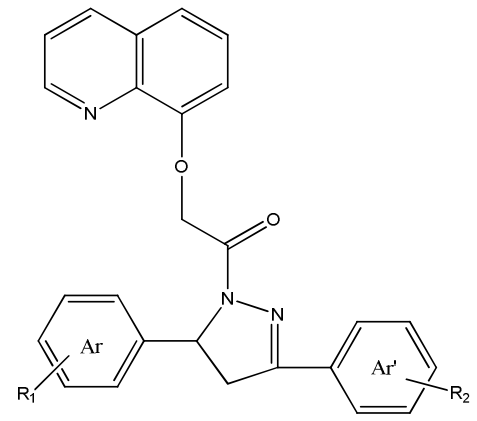

Figure 1. Structure of the compounds 7a-o (Refer Table 1) 


\section{Characterization data of 7a-o}

\section{1-(3,5-Diphenyl-4,5-dihydro-1H-pyrazol-1-yl)-2-(quinolin-8-yloxy)ethanone 7a}

Recrystallised from ethanol as yellow solid in $92 \%$ yield. m.p. $86-88^{\circ} \mathrm{C}$. IR $(\mathrm{KBr}) v_{\max }: 3074$ (C-H stretch in aromatics), $2935\left(\mathrm{C}-\mathrm{H}\right.$ stretch in $\left.\mathrm{CH}_{3} / \mathrm{CH}_{2}\right), 1672(\mathrm{C}=\mathrm{O}$ stretch $), 1632(\mathrm{C}=\mathrm{N}$ stretch), 1349 (C-N stretch), 1164, $1061\left(\mathrm{sp}^{2} / \mathrm{sp}^{3} \mathrm{C}-\mathrm{O}\right.$ stretch) $\mathrm{cm}^{-1}$.

${ }^{1} \mathrm{H}$ NMR (DMSO-d $\left.\mathrm{d}_{6}\right): \delta 4.92\left(\mathrm{~s}, 2 \mathrm{H}, \mathrm{OCH}_{2}\right), 3.16-3.21(\mathrm{dd}, \mathrm{J}=5.2,12.4,4.8 \mathrm{~Hz}, 1 \mathrm{H}$, pyrazole- $\left.\mathrm{H}_{4}\right), 3.74-3.81\left(\mathrm{dd}, \mathrm{J}=12,5.6 .12 .4,1 \mathrm{H}\right.$, pyrazole- $\left.\mathrm{H}_{4}\right), 5.65-5.69$ (dd, $\mathrm{J}=12,7.2$, $12.4 \mathrm{~Hz}, 1 \mathrm{H}$, pyrazole- $\left.\mathrm{H}_{5}\right), 7.30-7.46(\mathrm{~m}, 5 \mathrm{H}, \mathrm{Ar}-\mathrm{H}), 7.40-7.62(\mathrm{~m}, 5 \mathrm{H}, \mathrm{Ar}-\mathrm{H}), 7.38(\mathrm{~d}, 1 \mathrm{H}$, quinoline $\left.-\mathrm{H}_{7}\right), 7.60\left(\mathrm{t}, 1 \mathrm{H}\right.$, quinoline- $\left.\mathrm{H}_{3}\right), 7.66\left(\mathrm{~d}, 1 \mathrm{H}\right.$, quinoline- $\left.\mathrm{H}_{5}\right), 7.76(\mathrm{t}, 1 \mathrm{H}$, quinoline$\left.\mathrm{H}_{6}\right), 8.32\left(\mathrm{~d}, 1 \mathrm{H}\right.$, quinoline- $\left.\mathrm{H}_{4}\right), 8.89\left(\mathrm{~d}, 1 \mathrm{H}\right.$, quinoline $\left.-\mathrm{H}_{2}\right) \mathrm{ppm}$.

${ }^{13} \mathrm{C}$ NMR (DMSO-d $\left.\mathrm{d}_{6}\right): \delta 39.2$ (pyrazole $\left.\mathrm{CH}_{2}\right), 66.8$ (pyrazole $\left.\mathrm{CH}\right), 67.5\left(\mathrm{OCH}_{2}\right), 112.5$, 121.7, 122.6, 123.6, 126.5, 126.6, 128.2, 128.6, 128.8, 130.1, 131.1, 136.1, 136.3, 139.5, 141.3, 152.9, 154.6 (aromatic carbons), 151.2 (pyrazole $\mathrm{C}$ ), $168.7(\mathrm{C}=\mathrm{O}) \mathrm{ppm}$. MS $\mathrm{m} / \mathrm{z}$ : found 407 $\left[\mathrm{M}^{+}\right]$; calcd. 407. Anal. $\mathrm{C}_{26} \mathrm{H}_{21} \mathrm{~N}_{3} \mathrm{O}_{2}$. Found C 75.98 (76.64), H 5.09 (5.19), N 10.18 (10.31).

\section{1-(3-(4-Hydroxyphenyl)-5-phenyl-4,5-dihydro-1H-pyrazol-1-yl)-2-(quinolin-8- yloxy)ethanone $\mathbf{7 b}$}

Recrystallised from ethanol as yellow solid in $88 \%$ yield. m.p. $172-174{ }^{\circ} \mathrm{C}$. IR $(\mathrm{KBr}) \mathrm{v}_{\max }$ : 3368 (O-H stretch), 3012 (C-H stretch in aromatics), 2968 (C-H stretch in $\left.\mathrm{CH}_{3} / \mathrm{CH}_{2}\right), 1663$ $\left(\mathrm{C}=\mathrm{O}\right.$ stretch), $1628(\mathrm{C}=\mathrm{N}$ stretch$), 1339(\mathrm{C}-\mathrm{N}$ stretch$), 1172,1017\left(\mathrm{sp}^{2} / \mathrm{sp}^{3} \mathrm{C}-\mathrm{O}\right.$ stretch $) \mathrm{cm}^{-1}$.

${ }^{1} \mathrm{H}$ NMR (DMSO-d $): \delta 4.95\left(\mathrm{~s}, 2 \mathrm{H}, \mathrm{OCH}_{2}\right), 3.19-3.24(\mathrm{dd}, \mathrm{J}=4.4,12.8,4.8 \mathrm{~Hz}, 1 \mathrm{H}$, pyrazole- $\left.\mathrm{H}_{4}\right), 3.78-3.85\left(\mathrm{dd}, \mathrm{J}=12.8,6.4,8.2 \mathrm{~Hz}, 1 \mathrm{H}\right.$, pyrazole- $\left.\mathrm{H}_{4}\right), 5.36(\mathrm{~s}, 1 \mathrm{H}, \mathrm{OH}), 5.65-$ $5.69\left(\mathrm{dd}, \mathrm{J}=4.0,6.8,4.4 \mathrm{~Hz}, 1 \mathrm{H}\right.$, pyrazole- $\left.\mathrm{H}_{5}\right), 7.24-7.48(\mathrm{~m}, 5 \mathrm{H}, \mathrm{Ar}-\mathrm{H}), 6.85,6.88(\mathrm{~d}, \mathrm{~J}$ $=10 \mathrm{~Hz}, 2 \mathrm{H}, \mathrm{Ar}-\mathrm{H}), 7.94 \& 7.96\left(\mathrm{~d}, \mathrm{~J}=8 \mathrm{~Hz}, 2 \mathrm{H}, \mathrm{Ar}{ }^{\prime}-\mathrm{H}\right), 7.41\left(\mathrm{~d}, 1 \mathrm{H}\right.$, quinoline- $\left.\mathrm{H}_{7}\right), 7.62$ $\left(\mathrm{t}, 1 \mathrm{H}\right.$, quinoline- $\left.\mathrm{H}_{3}\right), 7.68\left(\mathrm{~d}, 1 \mathrm{H}\right.$, quinoline- $\left.\mathrm{H}_{5}\right), 7.79\left(\mathrm{t}, 1 \mathrm{H}\right.$, quinoline- $\left.\mathrm{H}_{6}\right), 8.31(\mathrm{~d}, 1 \mathrm{H}$, quinoline- $\left.\mathrm{H}_{4}\right), 8.86\left(\mathrm{~d}, 1 \mathrm{H}\right.$, quinoline- $\left.\mathrm{H}_{2}\right) \mathrm{ppm}$.

${ }^{13} \mathrm{C}$ NMR (DMSO-d $\left.{ }_{6}\right): \delta 39.4$ (pyrazole $\left.\mathrm{CH}_{2}\right), 66.6$ (pyrazole $\left.\mathrm{CH}\right), 67.8\left(\mathrm{OCH}_{2}\right), 111.8$, $115.4,121.6,122.5,123.8,126.5,126.6,128.5,128.6,128.8,130.0,135.9,140.2,141.3$, 151.9, 155.1, 157.8 (aromatic carbons), 151.4 (pyrazole C), $168.4(\mathrm{C}=\mathrm{O}) \mathrm{ppm}$. MS m/z : found $423\left[\mathrm{M}^{+}\right]$; calcd. 423. Anal. $\mathrm{C}_{26} \mathrm{H}_{21} \mathrm{~N}_{3} \mathrm{O}_{3}$. Found $\mathrm{C} 73.13$ (73.74), $\mathrm{H} 4.92$ (5.00), N 9.74 (9.92).

\section{1-(3-(2-Hydroxyphenyl)-5-phenyl-4,5-dihydro-1H-pyrazol-1-yl)-2-(quinolin-8-} yloxy)ethanone $7 \mathrm{c}$

Recrystallised from ethanol as lemon yellow solid in $90 \%$ yield. m.p. $70-72{ }^{\circ} \mathrm{C}$. IR $(\mathrm{KBr}) v_{\max }$ : 3392 (O-H stretch), 3021 (C-H stretch in aromatics), 2953 (C-H stretch in $\left.\mathrm{CH}_{3} / \mathrm{CH}_{2}\right), 1672(\mathrm{C}=\mathrm{O}$ stretch $), 1634(\mathrm{C}=\mathrm{N}$ stretch $), 1343(\mathrm{C}-\mathrm{N}$ stretch$), 1163,1033\left(\mathrm{sp}^{2} / \mathrm{sp}^{3}\right.$ $\mathrm{C}-\mathrm{O}$ stretch) $\mathrm{cm}^{-1}$.

${ }^{1} \mathrm{H}$ NMR (DMSO-d $\left.\mathrm{d}_{6}\right): \delta 4.91\left(\mathrm{~s}, 2 \mathrm{H}, \mathrm{OCH}_{2}\right), 3.11-3.16(\mathrm{dd}, \mathrm{J}=5.2,12.4,5.6 \mathrm{~Hz}, 1 \mathrm{H}$, pyrazole- $\left.\mathrm{H}_{4}\right), 3.69-3.76\left(\mathrm{dd}, \mathrm{J}=12,5.6,12.4 \mathrm{~Hz}, 1 \mathrm{H}\right.$, pyrazole- $\left.\mathrm{H}_{4}\right), 5.38(\mathrm{~s}, 1 \mathrm{H}, \mathrm{OH}), 5.58-$ $5.62\left(\mathrm{dd}, \mathrm{J}=4.4,7.2,4.8 \mathrm{~Hz}, 1 \mathrm{H}\right.$, pyrazole- $\left.\mathrm{H}_{5}\right), 7.31-7.41(\mathrm{~m}, 5 \mathrm{H}, \mathrm{Ar}-\mathrm{H}), 6.98-7.56(\mathrm{~m}, 4 \mathrm{H}$, Ar'-H), $7.46\left(\mathrm{~d}, 1 \mathrm{H}\right.$, quinoline- $\left.\mathrm{H}_{7}\right), 7.61\left(\mathrm{t}, 1 \mathrm{H}\right.$, quinoline- $\left.\mathrm{H}_{3}\right), 7.65\left(\mathrm{~d}, 1 \mathrm{H}\right.$, quinoline- $\left.\mathrm{H}_{5}\right)$, $7.74\left(\mathrm{t}, 1 \mathrm{H}\right.$, quinoline- $\left.\mathrm{H}_{6}\right), 8.35\left(\mathrm{~d}, 1 \mathrm{H}\right.$, quinoline- $\left.\mathrm{H}_{4}\right), 8.87\left(\mathrm{~d}, 1 \mathrm{H}\right.$, quinoline- $\left.\mathrm{H}_{2}\right) \mathrm{ppm}$.

${ }^{13} \mathrm{C}$ NMR (DMSO-d 6 ): $\delta 40.4$ (pyrazole $\left.\mathrm{CH}_{2}\right), 66.3$ (pyrazole $\left.\mathrm{CH}\right), 67.3\left(\mathrm{OCH}_{2}\right), 111.4$, 117.6, 118.6, 121.5, 121.6, 122.5, 123.5, 126.5, 126.8, 129.0, 130.2, 132.2, 132.3, 135.8, 140.1, 
141.6, 152.8, 155.2, 161.41 (aromatic carbons), 151.4 (pyrazole $\mathrm{C}$ ), $168.5(\mathrm{C}=\mathrm{O}) \mathrm{ppm}$. MS $\mathrm{m} / \mathrm{z}$ : found $423\left[\mathrm{M}^{+}\right]$; calcd. 423. Anal. $\mathrm{C}_{26} \mathrm{H}_{21} \mathrm{~N}_{3} \mathrm{O}_{3}$. Found C 73.18 (73.74), $\mathrm{H} 4.91$ (5.00), N $9.76(9.92)$.

\section{1-(3-(4-Chlorophenyl)-5-phenyl-4,5-dihydro-1H-pyrazol-1-yl)-2-(quinolin-8-yloxy) ethanone $7 d$}

Recrystallised from ethanol as cream solid in $94 \%$ yield. m.p. $124-126{ }^{\circ} \mathrm{C}$. IR $(\mathrm{KBr}) v_{\max }$ : 3068 (C-H stretch in aromatics), $2865\left(\mathrm{C}-\mathrm{H}\right.$ stretch in $\left.\mathrm{CH}_{3} / \mathrm{CH}_{2}\right), 1665(\mathrm{C}=\mathrm{O}$ stretch$), 1628$ $\left(\mathrm{C}=\mathrm{N}\right.$ stretch), 1352 (C-N stretch), 1193, 1017 ( $\mathrm{sp}^{2} / \mathrm{sp}^{3} \mathrm{C}-\mathrm{O}$ stretch), 1082 (C-Cl stretch in aromatics) $\mathrm{cm}^{-1}$.

${ }^{1} \mathrm{H}$ NMR (DMSO-d $): \delta 4.95\left(\mathrm{~s}, 2 \mathrm{H}, \mathrm{OCH}_{2}\right), 3.08-3.13(\mathrm{dd}, \mathrm{J}=4.0,12.4,4.4 \mathrm{~Hz}, 1 \mathrm{H}$, pyrazole- $\left.\mathrm{H}_{4}\right), 3.67-3.74\left(\mathrm{dd}, \mathrm{J}=12.0,4.4,12.4 \mathrm{~Hz}, 1 \mathrm{H}\right.$, pyrazole- $\left.\mathrm{H}_{4}\right), 5.60-5.64(\mathrm{dd}, \mathrm{J}=4.8$, 7.2, $5.2 \mathrm{~Hz}, 1 \mathrm{H}$, pyrazole- $\left.\mathrm{H}_{5}\right), 7.33-7.44(\mathrm{~m}, 5 \mathrm{H}, \mathrm{Ar}-\mathrm{H}), 7.50,7.53(\mathrm{~d}, \mathrm{~J}=8.8 \mathrm{~Hz}, 2 \mathrm{H}, \mathrm{Ar}$ $\mathrm{H}), 7.92,7.94(\mathrm{~d}, \mathrm{~J}=8.8 \mathrm{~Hz}, 2 \mathrm{H}, \mathrm{Ar}-\mathrm{H}), 7.48\left(\mathrm{~d}, 1 \mathrm{H}\right.$, quinoline- $\left.\mathrm{H}_{7}\right), 7.64(\mathrm{t}, 1 \mathrm{H}$, quinoline$\left.\mathrm{H}_{3}\right), 7.69\left(\mathrm{~d}, 1 \mathrm{H}\right.$, quinoline- $\left.\mathrm{H}_{5}\right), 7.76\left(\mathrm{t}, 1 \mathrm{H}\right.$, quinoline- $\left.\mathrm{H}_{6}\right), 8.32\left(\mathrm{~d}, 1 \mathrm{H}\right.$, quinoline- $\left.\mathrm{H}_{4}\right), 8.92$ (d, $1 \mathrm{H}$, quinoline- $\left.\mathrm{H}_{2}\right) \mathrm{ppm}$.

${ }^{13} \mathrm{C}$ NMR (DMSO-d $\left.\mathrm{d}_{6}\right): \delta 39.8$ (pyrazole $\left.\mathrm{CH}_{2}\right), 66.5$ (pyrazole $\left.\mathrm{CH}\right), 66.8\left(\mathrm{OCH}_{2}\right), 112.6$, $121.6,122.5,123.8,125.9,127.0,128.1,128.5,128.8,129.9,134.6,135.9,136.8,139.8$, 141.6, 150.2, 155.1 (aromatic carbons), 151.4 (pyrazole C), $169.3(\mathrm{C}=\mathrm{O}) \mathrm{ppm} . \mathrm{MS} \mathrm{m} / z$ : found $441\left[\mathrm{M}^{+}\right]$; calcd. 441. Anal. $\mathrm{C}_{26} \mathrm{H}_{20} \mathrm{ClN}_{3} \mathrm{O}_{2}$. Found $\mathrm{C} 70.28$ (70.67), $\mathrm{H} 4.45$ (4.56), N $9.38(9.51)$.

\section{1-(3-(4-Nitrophenyl)-5-phenyl-4,5-dihydro-1H-pyrazol-1-yl)-2-(quinolin-8-yloxy) ethanone $7 e$}

Recrystallised from ethanol as orange solid in $93 \%$ yield. m.p. $94-96^{\circ} \mathrm{C}$. IR $(\mathrm{KBr}) v_{\max }: 3060$ (C-H stretch in aromatics), 2924 (C-H stretch in $\left.\mathrm{CH}_{3} / \mathrm{CH}_{2}\right), 1665(\mathrm{C}=\mathrm{O}$ stretch), $1522(\mathrm{~N}-\mathrm{O}$ stretch), 1342 (C-N stretch), 1177, $1032\left(\mathrm{sp}^{2} / \mathrm{sp}^{3} \mathrm{C}-\mathrm{O}\right.$ stretch) $\mathrm{cm}^{-1}$.

${ }^{1} \mathrm{H}$ NMR (DMSO-d $\left.\mathrm{d}_{6}\right): \delta 4.94\left(\mathrm{~s}, 2 \mathrm{H}, \mathrm{OCH}_{2}\right), 3.17-3.23(\mathrm{dd}, \mathrm{J}=5.2,12.4,5.6 \mathrm{~Hz}, 1 \mathrm{H}$, pyrazole- $\left.\mathrm{H}_{4}\right), 3.76-3.83\left(\mathrm{dd}, \mathrm{J}=12.4,5.6,12.8 \mathrm{~Hz}, 1 \mathrm{H}\right.$, pyrazole- $\left.\mathrm{H}_{4}\right), 5.64-5.68(\mathrm{dd}, \mathrm{J}=4.0$, $6.8,4.4 \mathrm{~Hz}, 1 \mathrm{H}$, pyrazole- $\left.\mathrm{H}_{5}\right), 7.29-7.47(\mathrm{~m}, 5 \mathrm{H}, \mathrm{Ar}-\mathrm{H}), 8.14,8.16\left(\mathrm{~d}, \mathrm{~J}=8.8 \mathrm{~Hz}, 2 \mathrm{H}, \mathrm{Ar}{ }^{\prime}-\mathrm{H}\right)$, $8.29,8.32(\mathrm{~d}, \mathrm{~J}=9.2 \mathrm{~Hz}, 2 \mathrm{H}, \mathrm{Ar}-\mathrm{H}), 7.51\left(\mathrm{~d}, 1 \mathrm{H}\right.$, quinoline- $\left.\mathrm{H}_{7}\right), 7.55\left(\mathrm{t}, 1 \mathrm{H}\right.$, quinoline- $\left.\mathrm{H}_{3}\right)$, $7.65\left(\mathrm{~d}, 1 \mathrm{H}\right.$, quinoline- $\left.\mathrm{H}_{5}\right), 7.79\left(\mathrm{t}, 1 \mathrm{H}\right.$, quinoline- $\left.\mathrm{H}_{6}\right), 8.35\left(\mathrm{~d}, 1 \mathrm{H}\right.$, quinoline- $\left.\mathrm{H}_{4}\right), 8.87(\mathrm{~d}$, $1 \mathrm{H}$, quinoline- $\left.\mathrm{H}_{2}\right) \mathrm{ppm}$.

${ }^{13} \mathrm{C}$ NMR (DMSO-d $\left.\mathrm{d}_{6}\right): \delta 40.1$ (pyrazole $\left.\mathrm{CH}_{2}\right), 66.6$ (pyrazole $\left.\mathrm{CH}\right), 67.2\left(\mathrm{OCH}_{2}\right), 111.9$, 118.9, 121.8, 123.5, 126.7, 126.8, 126.9, 127.6, 128.6, 130.2, 136.0, 140.2, 141.8, 142.7, 149.9, 150.3, 154.8 (aromatic carbons), 151.1 (pyrazole C), $168.9(\mathrm{C}=\mathrm{O}) \mathrm{ppm}$. MS m/z : found $452\left[\mathrm{M}^{+}\right]$; calcd. 452. Anal. $\mathrm{C}_{26} \mathrm{H}_{20} \mathrm{~N}_{4} \mathrm{O}_{4}$. Found $\mathrm{C} 68.54$ (69.02), $\mathrm{H} 4.39$ (4.46), $\mathrm{N}$ 12.27 (12.38).

1-(5-(4-Methoxyphenyl)-3-phenyl-4,5-dihydro-1H-pyrazol-1-yl)-2-(quinolin-8yloxy)ethanone $7 f$

Recrystallised from ethanol as light brown solid in $91 \%$ yield. m.p. $126-128{ }^{\circ} \mathrm{C}$. IR $(\mathrm{KBr}) v_{\max }: 3082\left(\mathrm{C}-\mathrm{H}\right.$ stretch in aromatics), $2996\left(\mathrm{C}-\mathrm{H}\right.$ stretch in $\left.\mathrm{CH}_{3} / \mathrm{CH}_{2}\right), 1668(\mathrm{C}=\mathrm{O}$ stretch), 1630 (C=N stretch), 1361 (C-N stretch), 1225, $1062\left(\mathrm{sp}^{2} / \mathrm{sp}^{3} \mathrm{C}-\mathrm{O}\right.$ stretch) $\mathrm{cm}^{-1}$.

${ }^{1} \mathrm{H}$ NMR (DMSO-d $\left.\mathrm{d}_{6}\right): \delta 3.86\left(\mathrm{~s}, 3 \mathrm{H}, \mathrm{OCH}_{3}\right), 4.92\left(\mathrm{~s}, 2 \mathrm{H}, \mathrm{OCH}_{2}\right), 3.12-3.17(\mathrm{dd}, \mathrm{J}=4.8$, $13.2,4.4 \mathrm{~Hz}, 1 \mathrm{H}$, pyrazole- $\left.\mathrm{H}_{4}\right), 3.78-3.85\left(\mathrm{dd}, \mathrm{J}=13.2,4.4 .13 .6,1 \mathrm{H}\right.$, pyrazole- $\left.\mathrm{H}_{4}\right), 5.66-$ $5.70\left(\mathrm{dd}, \mathrm{J}=4.4,7.2,4.8 \mathrm{~Hz}, 1 \mathrm{H}\right.$, pyrazole- $\left.\mathrm{H}_{5}\right), 6.89,6.91(\mathrm{~d}, \mathrm{~J}=8 \mathrm{~Hz}, 2 \mathrm{H}, \mathrm{Ar}-\mathrm{H}), 7.12,7.14$ 
$(\mathrm{d}, \mathrm{J}=8 \mathrm{~Hz}, 2 \mathrm{H}, \mathrm{Ar}-\mathrm{H}), 7.54-7.69(\mathrm{~m}, 5 \mathrm{H}, \mathrm{Ar}-\mathrm{H}), 7.51\left(\mathrm{~d}, 1 \mathrm{H}\right.$, quinoline- $\left.\mathrm{H}_{7}\right), 7.58(\mathrm{t}, 1 \mathrm{H}$, quinoline- $\left.\mathrm{H}_{3}\right), 7.63\left(\mathrm{~d}, 1 \mathrm{H}\right.$, quinoline- $\left.\mathrm{H}_{5}\right), 7.78\left(\mathrm{t}, 1 \mathrm{H}\right.$, quinoline- $\left.\mathrm{H}_{6}\right), 8.31(\mathrm{~d}, 1 \mathrm{H}$, quinoline$\left.\mathrm{H}_{4}\right), 8.82\left(\mathrm{~d}, 1 \mathrm{H}\right.$, quinoline- $\left.\mathrm{H}_{2}\right) \mathrm{ppm}$.

${ }^{13} \mathrm{C}$ NMR (DMSO-d $\left.{ }_{6}\right): \delta 39.7$ (pyrazole $\left.\mathrm{CH}_{2}\right), 55.9\left(\mathrm{OCH}_{3}\right), 66.4$ (pyrazole $\left.\mathrm{CH}\right), 66.9$ $\left(\mathrm{OCH}_{2}\right), 112.8,114.2,121.6,122.4,123.5,126.7,128.3,128.6,129.8$ (2), 135.8, 136.8, 139.7, 139.8, 151.8, 154.3, 158.6 (aromatic carbons), 151.9 (pyrazole $\mathrm{C}$ ), $168.5(\mathrm{C}=\mathrm{O}) \mathrm{ppm}$. MS m/z: found $437\left[\mathrm{M}^{+}\right]$; calcd. 437. Anal. $\mathrm{C}_{27} \mathrm{H}_{23} \mathrm{~N}_{3} \mathrm{O}_{3}$. Found $\mathrm{C} 73.74$ (74.12), H 5.24 (5.30), N 9.44 (9.60).

1-(3-(4-Hydroxyphenyl)-5-(4-methoxyphenyl)-4,5-dihydro-1H-pyrazol-1-yl)-2(quinolin-8-yloxy)ethanone $\mathbf{7 g}$

Recrystallised from ethanol as orange solid in $86 \%$ yield. m.p. $226-228{ }^{\circ} \mathrm{C}$. IR $(\mathrm{KBr}) v_{\max }$ : 3373 (O-H stretch), 2988 (C-H stretch in aromatics), 2913 (C-H stretch in $\left.\mathrm{CH}_{3} / \mathrm{CH}_{2}\right), 1672$ $\left(\mathrm{C}=\mathrm{O}\right.$ stretch), 1631 (C=N stretch), 1598 (C=C stretch), 1185, $1042\left(\mathrm{sp}^{2} / \mathrm{sp}^{3} \mathrm{C}-\mathrm{O}\right.$ stretch $) \mathrm{cm}^{-1}$.

${ }^{1} \mathrm{H}$ NMR (DMSO-d 6 ) : $\delta 3.87\left(\mathrm{~s}, 3 \mathrm{H}, \mathrm{OCH}_{3}\right), 4.90\left(\mathrm{~s}, 2 \mathrm{H}, \mathrm{OCH}_{2}\right), 3.15-3.20(\mathrm{dd}, \mathrm{J}=4.8$, $12.4,4.4 \mathrm{~Hz}, 1 \mathrm{H}$, pyrazole- $\left.\mathrm{H}_{4}\right), 3.70-3.77\left(\mathrm{dd}, \mathrm{J}=12.0,4.8,12.4 \mathrm{~Hz}, 1 \mathrm{H}\right.$, pyrazole- $\left.\mathrm{H}_{4}\right), 5.39(\mathrm{~s}$, $1 \mathrm{H}, \mathrm{OH}), 5.61-5.65\left(\mathrm{dd}, \mathrm{J}=4.0,6.8,4.4 \mathrm{~Hz}, 1 \mathrm{H}\right.$, pyrazole- $\left.\mathrm{H}_{5}\right), 6.85,6.87(\mathrm{~d}, \mathrm{~J}=8 \mathrm{~Hz}, 2 \mathrm{H}, \mathrm{Ar}-$ H), 7.08, $7.10(\mathrm{~d}, \mathrm{~J}=8 \mathrm{~Hz}, 2 \mathrm{H}, \mathrm{Ar}-\mathrm{H}), 6.81,6.84(\mathrm{~d}, \mathrm{~J}=12 \mathrm{~Hz}, 2 \mathrm{H}, \mathrm{Ar}-\mathrm{H}), 7.92,7.94$ (d, J = 8 $\mathrm{Hz}, 2 \mathrm{H}, \mathrm{Ar}-\mathrm{H}), 7.54\left(\mathrm{~d}, 1 \mathrm{H}\right.$, quinoline- $\left.\mathrm{H}_{7}\right), 7.61\left(\mathrm{t}, 1 \mathrm{H}\right.$, quinoline- $\left.\mathrm{H}_{3}\right), 7.65(\mathrm{~d}, 1 \mathrm{H}$, quinoline$\left.\mathrm{H}_{5}\right), 7.76\left(\mathrm{t}, 1 \mathrm{H}\right.$, quinoline- $\left.\mathrm{H}_{6}\right), 8.36\left(\mathrm{~d}, 1 \mathrm{H}\right.$, quinoline- $\left.\mathrm{H}_{4}\right), 8.80\left(\mathrm{~d}, 1 \mathrm{H}\right.$, quinoline- $\left.\mathrm{H}_{2}\right) \mathrm{ppm}$.

${ }^{13} \mathrm{C}$ NMR (DMSO-d $\left.{ }_{6}\right): \delta 40.1$ (pyrazole $\left.\mathrm{CH}_{2}\right), 55.6\left(\mathrm{OCH}_{3}\right), 66.5$ (pyrazole $\left.\mathrm{CH}\right), 67.4$ $\left(\mathrm{OCH}_{2}\right), 111.8,114.3,116.8,121.5,122.7,123.8,126.7,128.9,129.3,129.9,134.8,135.9$, 140.5, 152.5, 154.4, 158.9, 160.2 (aromatic carbons), 151.9 (pyrazole $\mathrm{C}$ ), $168.9(\mathrm{C}=\mathrm{O}) \mathrm{ppm}$. MS $m / z$ : found $453\left[\mathrm{M}^{+}\right]$; calcd. 453. Anal. $\mathrm{C}_{27} \mathrm{H}_{23} \mathrm{~N}_{3} \mathrm{O}_{4}$. Found $\mathrm{C} 70.98$ (71.51), H 5.06 (5.11), N 9.11 (9.27).

\section{1-(3-(2-Hydroxyphenyl)-5-(4-methoxyphenyl)-4,5-dihydro-1H-pyrazol-1-yl)-2-} (quinolin-8-yloxy)ethanone $7 \boldsymbol{h}$

Recrystallised from ethanol as lemon yellow solid in $92 \%$ yield. m.p. 136-138 ${ }^{\circ} \mathrm{C}$. IR $(\mathrm{KBr}) v_{\max }$ : $3347\left(\mathrm{O}-\mathrm{H}\right.$ stretch), $2987\left(\mathrm{C}-\mathrm{H}\right.$ stretch in $\left.\mathrm{CH}_{3} / \mathrm{CH}_{2}\right), 1682(\mathrm{C}=\mathrm{O}$ stretch$), 1638$ $\left(\mathrm{C}=\mathrm{N}\right.$ stretch), $1604(\mathrm{C}=\mathrm{C}$ stretch in aromatics $), 1176,1029\left(\mathrm{sp}^{2} / \mathrm{sp}^{3} \mathrm{C}-\mathrm{O}\right.$ stretch $) \mathrm{cm}^{-1}$.

${ }^{1} \mathrm{H}$ NMR $\left(\mathrm{DMSO}_{6}\right): \delta 3.87\left(\mathrm{~s}, 3 \mathrm{H}, \mathrm{OCH}_{3}\right), 4.88\left(\mathrm{~s}, 2 \mathrm{H}, \mathrm{OCH}_{2}\right), 3.10-3.15(\mathrm{dd}, \mathrm{J}=5.2$, $12.4,5.6 \mathrm{~Hz}, 1 \mathrm{H}$, pyrazole- $\left.\mathrm{H}_{4}\right), 3.71-3.78\left(\mathrm{dd}, \mathrm{J}=12.0,5.6,12.4 \mathrm{~Hz}, 1 \mathrm{H}\right.$, pyrazole- $\left.\mathrm{H}_{4}\right), 5.37$ (s, 1H, OH), 5.57-5.61 (dd, J = 4.4, 6.8, 4.0 Hz, 1H, pyrazole- $\left.\mathrm{H}_{5}\right), 6.86,6.88(\mathrm{~d}, 2 \mathrm{H}, \mathrm{Ar}-\mathrm{H})$, 7.21, $7.23(\mathrm{~d}, 2 \mathrm{H}, \mathrm{Ar}-\mathrm{H}), 6.95-7.48\left(\mathrm{~m}, 4 \mathrm{H}, \mathrm{Ar}{ }^{\prime}-\mathrm{H}\right), 7.52\left(\mathrm{~d}, 1 \mathrm{H}\right.$, quinoline- $\left.\mathrm{H}_{7}\right), 7.62(\mathrm{t}, 1 \mathrm{H}$, quinoline- $\left.\mathrm{H}_{3}\right), 7.66\left(\mathrm{~d}, 1 \mathrm{H}\right.$, quinoline- $\left.\mathrm{H}_{5}\right), 7.78\left(\mathrm{t}, 1 \mathrm{H}\right.$, quinoline- $\left.\mathrm{H}_{6}\right), 8.32(\mathrm{~d}, 1 \mathrm{H}$, quinoline- $\left.\mathrm{H}_{4}\right), 8.81\left(\mathrm{~d}, 1 \mathrm{H}\right.$, quinoline- $\left.\mathrm{H}_{2}\right) \mathrm{ppm}$.

${ }^{13} \mathrm{C}$ NMR (DMSO-d $\left.{ }_{6}\right): \delta 40.5$ (pyrazole $\left.\mathrm{CH}_{2}\right), 55.7\left(\mathrm{OCH}_{3}\right), 66.4$ (pyrazole $\left.\mathrm{CH}\right), 66.9$ $\left(\mathrm{OCH}_{2}\right), 112.8,114.4,117.9,118.8,121.3,121.6,122.8,123.7,126.4,129.9,131.9,132.5$, $133.8,135.8,138.9,150.2,154.9,158.2,162.6$ (aromatic carbons), 151.6 (pyrazole C), $168.8(\mathrm{C}=\mathrm{O}) \mathrm{ppm}$. MS $\mathrm{m} / \mathrm{z}$ : found $453\left[\mathrm{M}^{+}\right]$; calcd. 453. Anal. $\mathrm{C}_{27} \mathrm{H}_{23} \mathrm{~N}_{3} \mathrm{O}_{4}$. Found $\mathrm{C} 71.12$ (71.51), H 5.02 (5.11), N 9.14 (9.27).

1-(3-(4-Chlorophenyl)-5-(4-methoxyphenyl)-4,5-dihydro-1H-pyrazol-1-yl)-2(quinolin-8-yloxy)ethanone $7 \boldsymbol{i}$

Recrystallised from ethanol as cream solid in $94 \%$ yield. m.p. $170-172{ }^{\circ} \mathrm{C}$. IR $(\mathrm{KBr}) v_{\max }$ : 3074 (C-H stretch in aromatics), 2932 (C-H stretch in $\left.\mathrm{CH}_{3} / \mathrm{CH}_{2}\right), 1658$ (C=O stretch), 1632 
$(\mathrm{C}=\mathrm{N}$ stretch$), 1606(\mathrm{C}=\mathrm{C}$ stretch in aromatics $), 1184,1028\left(\mathrm{sp}^{2} / \mathrm{sp}^{3} \mathrm{C}-\mathrm{O}\right.$ stretch $), 1076$ (C-Cl stretch in aromatics) $\mathrm{cm}^{-1}$.

${ }^{1} \mathrm{H}$ NMR (DMSO-d $): \delta 3.86\left(\mathrm{~s}, 3 \mathrm{H}, \mathrm{OCH}_{3}\right), 4.92\left(\mathrm{~s}, 2 \mathrm{H}, \mathrm{OCH}_{2}\right), 3.21-3.26(\mathrm{dd}, \mathrm{J}=$ 4.8, 12.4, $4.4 \mathrm{~Hz}, 1 \mathrm{H}$, pyrazole- $\mathrm{H}_{4}$ ), 3.84-3.91 (dd, $\mathrm{J}=12.0,4.8,12.4 \mathrm{~Hz}, 1 \mathrm{H}$, pyrazole$\left.\mathrm{H}_{4}\right), 5.80-5.84\left(\mathrm{dd}, \mathrm{J}=4.4,7.2,4.8 \mathrm{~Hz}, 1 \mathrm{H}\right.$ pyrazole- $\left.\mathrm{H}_{5}\right), 6.80 \& 6.82(\mathrm{~d}, 2 \mathrm{H}, \mathrm{Ar}-\mathrm{H}), 7.11$ \& $7.13(\mathrm{~d}, 2 \mathrm{H}, \operatorname{Ar}-\mathrm{H}), 7.45,7.47\left(\mathrm{~d}, \mathrm{~J}=8.0 \mathrm{~Hz}, 2 \mathrm{H}, \mathrm{Ar}{ }^{\prime}-\mathrm{H}\right), 7.95,7.97(\mathrm{~d}, \mathrm{~J}=8.0 \mathrm{~Hz}$, $2 \mathrm{H}, \quad \mathrm{Ar} \cdot-\mathrm{H}), 7.49\left(\mathrm{~d}, 1 \mathrm{H}\right.$, quinoline- $\left.\mathrm{H}_{7}\right), 7.56\left(\mathrm{t}, 1 \mathrm{H}\right.$, quinoline- $\left.\mathrm{H}_{3}\right), 7.65(\mathrm{~d}, 1 \mathrm{H}$, quinoline- $\left.\mathrm{H}_{5}\right), 7.76\left(\mathrm{t}, 1 \mathrm{H}\right.$, quinoline- $\left.\mathrm{H}_{6}\right), 8.35\left(\mathrm{~d}, 1 \mathrm{H}\right.$, quinoline- $\left.\mathrm{H}_{4}\right), 8.84(\mathrm{~d}, 1 \mathrm{H}$, quinoline- $\mathrm{H}_{2}$ ) ppm.

${ }^{13} \mathrm{C}$ NMR (DMSO-d $\left.{ }_{6}\right): \delta 39.8\left(\right.$ pyrazole $\left.\mathrm{CH}_{2}\right), 55.6\left(\mathrm{OCH}_{3}\right), 66.4$ (pyrazole $\left.\mathrm{CH}\right), 66.8$ $\left(\mathrm{OCH}_{2}\right), 113.2,114.3,121.4,122.6,123.8,126.4,128.4,128.9,130.1,134.4,134.7,135.4$, 136.5, 138.6, 151.5, 154.9, 158.4 (aromatic carbons), 151.2 (pyrazole $\mathrm{C}$ ), $168.5(\mathrm{C}=\mathrm{O}) \mathrm{ppm}$. MS $m / z$ : found $471\left[\mathrm{M}^{+}\right]$; calcd. 471. Anal. $\mathrm{C}_{27} \mathrm{H}_{22} \mathrm{ClN}_{3} \mathrm{O}_{3}$. Found $\mathrm{C} 68.33$ (68.71), $\mathrm{H} 4.61$ (4.70), N 8.75 (8.90).

\section{1-(5-(4-methoxyphenyl)-3-(4-nitrophenyl)-4,5-dihydro-1H-pyrazol-1-yl)-2-} (quinolin-8-yloxy)ethanone $7 \boldsymbol{j}$

Recrystallised from ethanol as light brown solid in 93\% yield. m.p. $128-130{ }^{\circ} \mathrm{C}$. IR $(\mathrm{KBr}) v_{\max }: 3065\left(\mathrm{C}-\mathrm{H}\right.$ stretch in aromatics), $2824\left(\mathrm{C}-\mathrm{H}\right.$ stretch in $\left.\mathrm{CH}_{3} / \mathrm{CH}_{2}\right), 1668(\mathrm{C}=\mathrm{O}$ stretch), $1636(\mathrm{C}=\mathrm{N}$ stretch), 1561 (N-O stretch), $1498(\mathrm{C}=\mathrm{C}$ stretch in aromatics), 1196, $1046\left(\mathrm{sp}^{2} / \mathrm{sp}^{3} \mathrm{C}-\mathrm{O}\right.$ stretch) $\mathrm{cm}^{-1}$.

${ }^{1} \mathrm{H}$ NMR $\left(\right.$ DMSO-d $\left._{6}\right): \delta 3.86\left(\mathrm{~s}, 3 \mathrm{H}, \mathrm{OCH}_{3}\right), 4.95\left(\mathrm{~s}, 2 \mathrm{H}, \mathrm{OCH}_{2}\right), 3.16-3.21(\mathrm{dd}, \mathrm{J}=4.8$, 13.2, $4.4 \mathrm{~Hz}, 1 \mathrm{H}$, pyrazole- $\left.\mathrm{H}_{4}\right), 3.77-3.86\left(\mathrm{dd}, \mathrm{J}=12.8,4.8,13.2 \mathrm{~Hz}, 1 \mathrm{H}\right.$, pyrazole- $\left.\mathrm{H}_{4}\right), 5.58-5.62$ (dd, J = 4.4, 7.2, $4.8 \mathrm{~Hz}, 1 \mathrm{H}$ pyrazole- $\left.\mathrm{H}_{5}\right), 6.84 \& 6.86(\mathrm{~d}, \mathrm{~J}=8.4 \mathrm{~Hz}, 2 \mathrm{H}, \mathrm{Ar}-\mathrm{H}), 7.14$ \& 7.16 (d, $\mathrm{J}=8.4 \mathrm{~Hz}, 2 \mathrm{H}, \mathrm{Ar}-\mathrm{H}), 8.12 \& 8.14\left(\mathrm{~d}, \mathrm{~J}=8.4 \mathrm{~Hz}, 2 \mathrm{H}, \mathrm{Ar}{ }^{\prime}-\mathrm{H}\right), 8.27 \& 8.29(\mathrm{~d}, \mathrm{~J}=8.4 \mathrm{~Hz}, 2 \mathrm{H}$, Ar'-H), $7.38\left(\mathrm{~d}, 1 \mathrm{H}\right.$, quinoline- $\left.\mathrm{H}_{7}\right), 7.61\left(\mathrm{t}, 1 \mathrm{H}\right.$, quinoline- $\left.\mathrm{H}_{3}\right), 7.62(\mathrm{~d}, \mathrm{~J}=8.4 \mathrm{~Hz}, 1 \mathrm{H}$, quinoline$\left.\mathrm{H}_{5}\right), 7.63\left(\mathrm{t}, 1 \mathrm{H}\right.$, quinoline- $\left.\mathrm{H}_{6}\right), 8.33\left(\mathrm{~d}, 1 \mathrm{H}\right.$, quinoline- $\left.\mathrm{H}_{4}\right), 8.86\left(\mathrm{~d}, 1 \mathrm{H}\right.$, quinoline- $\left.\mathrm{H}_{2}\right) \mathrm{ppm}$.

${ }^{13} \mathrm{C}$ NMR (DMSO-d $\left.{ }_{6}\right): \delta 40.1\left(\right.$ pyrazole $\left.\mathrm{CH}_{2}\right), 55.9\left(\mathrm{OCH}_{3}\right), 66.4$ (pyrazole $\left.\mathrm{CH}\right), 67.2$ $\left(\mathrm{OCH}_{2}\right), 106.8,114.5,118.6,121.6,124.3,126.8,127.1,127.6,130.5,134.2,136.8,140.3$, 142.3, 149.4, 150.2, 156.1, 158.9 (aromatic carbons), 151.6 (pyrazole $\mathrm{C}), 170.2(\mathrm{C}=\mathrm{O}) \mathrm{ppm}$. MS $m / z$ : found $482\left[\mathrm{M}^{+}\right]$; calcd. 482. Anal. $\mathrm{C}_{27} \mathrm{H}_{22} \mathrm{~N}_{4} \mathrm{O}_{5}$. Found $\mathrm{C} 66.88$ (67.21), H 4.53 (4.60), N 11.49 (11.61).

\section{1-(5-(2-Hydroxyphenyl)-3-phenyl-4,5-dihydro-1H-pyrazol-1-yl)-2-(quinolin-8- yloxy)ethanone $7 \boldsymbol{k}$}

Recrystallised from ethanol as yellow solid in $72 \%$ yield. m.p. $100-102{ }^{\circ} \mathrm{C}$. IR $(\mathrm{KBr}) v_{\max }$ : 3410 (O-H stretch), 3071 (C-H stretch in aromatics), 2865 (C-H stretch in $\left.\mathrm{CH}_{3} / \mathrm{CH}_{2}\right), 1675$ $(\mathrm{C}=\mathrm{O}$ stretch $), 1632(\mathrm{C}=\mathrm{N}$ stretch $), 1486(\mathrm{C}=\mathrm{C}$ stretch in aromatics $), 1186,1042\left(\mathrm{sp}^{2} / \mathrm{sp}^{3} \mathrm{C}-\right.$ O stretch) $\mathrm{cm}^{-1}$.

${ }^{1} \mathrm{H}$ NMR (DMSO-d $): \delta 4.94\left(\mathrm{~s}, 2 \mathrm{H}, \mathrm{OCH}_{2}\right), 3.18-3.23(\mathrm{dd}, \mathrm{J}=5.2,13.2,5.6 \mathrm{~Hz}, 1 \mathrm{H}$, pyrazole- $\left.\mathrm{H}_{4}\right), 3.78-3.85\left(\mathrm{dd}, \mathrm{J}=12.8,5.6 .13 .2,1 \mathrm{H}\right.$, pyrazole- $\left.\mathrm{H}_{4}\right), 5.38(\mathrm{~s}, 1 \mathrm{H}, \mathrm{OH}), 5.70-$ $5.74\left(\mathrm{dd}, \mathrm{J}=4.0,6.8,4.4 \mathrm{~Hz}, 1 \mathrm{H}\right.$, pyrazole- $\left.\mathrm{H}_{5}\right), 6.94-7.10(\mathrm{~m}, 4 \mathrm{H}, \mathrm{Ar}-\mathrm{H}), 7.48-7.68(\mathrm{~m}, 5 \mathrm{H}$, $\mathrm{Ar}$ '- $\mathrm{H}), 7.54\left(\mathrm{~d}, 1 \mathrm{H}\right.$, quinoline- $\left.\mathrm{H}_{7}\right), 7.58\left(\mathrm{t}, 1 \mathrm{H}\right.$, quinoline- $\left.\mathrm{H}_{3}\right), 7.60\left(\mathrm{~d}, 1 \mathrm{H}\right.$, quinoline- $\left.\mathrm{H}_{5}\right)$, $7.65\left(\mathrm{t}, 1 \mathrm{H}\right.$, quinoline- $\left.\mathrm{H}_{6}\right), 8.32\left(\mathrm{~d}, 1 \mathrm{H}\right.$, quinoline- $\left.\mathrm{H}_{4}\right), 8.86\left(\mathrm{~d}, 1 \mathrm{H}\right.$, quinoline- $\left.\mathrm{H}_{2}\right) \mathrm{ppm}$.

${ }^{13} \mathrm{C}$ NMR (DMSO-d $\mathrm{d}_{6}$ ): $\delta 40.6$ (pyrazole $\left.\mathrm{CH}_{2}\right), 58.3$ (pyrazole $\left.\mathrm{CH}\right), 66.8\left(\mathrm{OCH}_{2}\right), 108.9$, 115.6, 117.8, 121.2, 121.9, 126.4, 126.7, 128.4, 128.6, 128.9, 130.1 (2), 131.2, 136.2, 136.5, 
140.3, 150.1, 154.8, 155.8 (aromatic carbons), 151.8 (pyrazole $\mathrm{C}$ ), $169.8(\mathrm{C}=\mathrm{O}) \mathrm{ppm}$. MS $\mathrm{m} / \mathrm{z}$ : found $423\left[\mathrm{M}^{+}\right]$; calcd. 423. Anal. $\mathrm{C}_{26} \mathrm{H}_{21} \mathrm{~N}_{3} \mathrm{O}_{3}$. Found $\mathrm{C} 73.32$ (73.74), $\mathrm{H} 4.95$ (5.00), N 9.77 (9.92).

\section{1-(5-(2-Hydroxyphenyl)-3-(4-hydroxyphenyl)-4,5-dihydro-1H-pyrazol-1-yl)-2-} (quinolin-8-yloxy)ethanone $7 \boldsymbol{l}$

Recrystallised from ethanol as yellow solid in $70 \%$ yield. m.p. $182-184{ }^{\circ} \mathrm{C}$. IR $(\mathrm{KBr}) \mathrm{v}_{\max }$ : 3315 (O-H stretch), 3024 (C-H stretch in aromatics), 2843 (C-H stretch in $\left.\mathrm{CH}_{3} / \mathrm{CH}_{2}\right), 1681$ $(\mathrm{C}=\mathrm{O}$ stretch $), 1628(\mathrm{C}=\mathrm{N}$ stretch $), 1480(\mathrm{C}=\mathrm{C}$ stretch in aromatics $), 1171,1034\left(\mathrm{sp}^{2} / \mathrm{sp}^{3} \mathrm{C}-\right.$ O stretch) $\mathrm{cm}^{-1}$.

${ }^{1} \mathrm{H}$ NMR (DMSO-d $): \delta 4.95\left(\mathrm{~s}, 2 \mathrm{H}, \mathrm{OCH}_{2}\right), 3.15-3.20(\mathrm{dd}, \mathrm{J}=4.4,12.4,4.0 \mathrm{~Hz}, 1 \mathrm{H}$, pyrazole- $\left.\mathrm{H}_{4}\right), 3.76-3.83\left(\mathrm{dd}, \mathrm{J}=12.0,4.0,12.4 \mathrm{~Hz}, 1 \mathrm{H}\right.$, pyrazole- $\left.\mathrm{H}_{4}\right), 5.39(\mathrm{~s}, 2 \mathrm{H}, \mathrm{OH})$, 5.67-5.71 (dd, J = 4.4, 7.2, 4.8 Hz, 1H, pyrazole- $\left.\mathrm{H}_{5}\right), 6.87-7.08(\mathrm{~m}, 4 \mathrm{H}, \mathrm{Ar}-\mathrm{H}), 6.80-6.83$ $\left(\mathrm{d}, \mathrm{J}=12 \mathrm{~Hz}, 2 \mathrm{H}, \mathrm{Ar} \mathbf{r}^{-\mathrm{H}}\right), 7.91,7.93(\mathrm{~d}, \mathrm{~J}=8 \mathrm{~Hz}, 2 \mathrm{H}, \mathrm{Ar}-\mathrm{H}), 7.51$ (d, 1H, quinoline- $\left.\mathrm{H}_{7}\right)$, $7.58\left(\mathrm{t}, 1 \mathrm{H}\right.$, quinoline- $\left.\mathrm{H}_{3}\right), 7.65\left(\mathrm{~d}, 1 \mathrm{H}\right.$, quinoline- $\left.\mathrm{H}_{5}\right), 7.76\left(\mathrm{t}, 1 \mathrm{H}\right.$, quinoline- $\left.\mathrm{H}_{6}\right), 8.34$ $\left(\mathrm{d}, 1 \mathrm{H}\right.$, quinoline- $\left.\mathrm{H}_{4}\right), 8.81\left(\mathrm{~d}, 1 \mathrm{H}\right.$, quinoline- $\left.\mathrm{H}_{2}\right) \mathrm{ppm}$.

${ }^{13} \mathrm{C}$ NMR (DMSO-d $): \delta 40.3$ (pyrazole $\left.\mathrm{CH}_{2}\right), 59.8$ (pyrazole $\left.\mathrm{CH}\right), 67.2\left(\mathrm{OCH}_{2}\right), 108.9$, $115.9,116.2,118.2,120.8,121.7,126.1,126.8,128.4,128.8,128.9,129.6,130.6,135.9$, 140.1, 148.2, 154.7, 155.8, 159.6 (aromatic carbons), 151.6 (pyrazole $\mathrm{C}$ ), $169.4(\mathrm{C}=\mathrm{O}) \mathrm{ppm}$. MS $m / z$ : found $439\left[\mathrm{M}^{+}\right]$; calcd. 439. Anal. $\mathrm{C}_{26} \mathrm{H}_{21} \mathrm{~N}_{3} \mathrm{O}_{4}$. Found $\mathrm{C} 70.67$ (71.06), H 4.76 (4.82), N 9.41 (9.56).

\section{1-(3,5-Bis(2-hydroxyphenyl)-4,5-dihydro-1H-pyrazol-1-yl)-2-(quinolin-8- yloxy)ethanone $7 \mathbf{m}$}

Recrystallised from ethanol as yellow solid in $65 \%$ yield. m.p. $150-152{ }^{\circ} \mathrm{C}$. IR $(\mathrm{KBr}) v_{\max }$ : 3318 (O-H stretch), 3041 (C-H stretch in aromatics), 2827 (C-H stretch in $\left.\mathrm{CH}_{3} / \mathrm{CH}_{2}\right), 1678$ $(\mathrm{C}=\mathrm{O}$ stretch$), 1641(\mathrm{C}=\mathrm{N}$ stretch$), 1493(\mathrm{C}=\mathrm{C}$ stretch in aromatics $), 1164,1039\left(\mathrm{sp}^{2} / \mathrm{sp}^{3}\right.$ C-O stretch) $\mathrm{cm}^{-1}$.

${ }^{1} \mathrm{H}$ NMR (DMSO-d $\left.{ }_{6}\right): \delta 4.86\left(\mathrm{~s}, 2 \mathrm{H}, \mathrm{OCH}_{2}\right), 3.14-3.19(\mathrm{dd}, \mathrm{J}=4.8,12.4,4.4 \mathrm{~Hz}, 1 \mathrm{H}$, pyrazole- $\left.\mathrm{H}_{4}\right), 3.80-3.87\left(\mathrm{dd}, \mathrm{J}=12.0,4.8,12.4 \mathrm{~Hz}, 1 \mathrm{H}\right.$, pyrazole- $\left.\mathrm{H}_{4}\right), 5.37(\mathrm{~s}, 2 \mathrm{H}, \mathrm{OH}), 5.73-$ $5.77\left(\mathrm{dd}, \mathrm{J}=4.4,7.2,4.8 \mathrm{~Hz}, 1 \mathrm{H}\right.$, pyrazole- $\left.\mathrm{H}_{5}\right), 6.88-7.10(\mathrm{~m}, 4 \mathrm{H}, \mathrm{Ar}-\mathrm{H}), 6.94-7.51(\mathrm{~m}, 4 \mathrm{H}$, Ar'-H), $7.48\left(\mathrm{~d}, 1 \mathrm{H}\right.$, quinoline- $\left.\mathrm{H}_{7}\right), 7.59\left(\mathrm{t}, 1 \mathrm{H}\right.$, quinoline- $\left.\mathrm{H}_{3}\right), 7.63\left(\mathrm{~d}, 1 \mathrm{H}\right.$, quinoline- $\left.\mathrm{H}_{5}\right), 7.75$ $\left(\mathrm{t}, 1 \mathrm{H}\right.$, quinoline- $\left.\mathrm{H}_{6}\right), 8.36\left(\mathrm{~d}, 1 \mathrm{H}\right.$, quinoline- $\left.\mathrm{H}_{4}\right), 8.84\left(\mathrm{~d}, 1 \mathrm{H}\right.$, quinoline- $\left.\mathrm{H}_{2}\right) \mathrm{ppm}$.

${ }^{13} \mathrm{C}$ NMR (DMSO-d $): \delta 40.5$ (pyrazole $\left.\mathrm{CH}_{2}\right), 59.2($ pyrazole $\mathrm{CH}), 67.2\left(\mathrm{OCH}_{2}\right), 108.1$, $115.9,117.5,118.2,118.6,121.3,121.5,121.7,126.4,126.7,128.2,130.1,130.5,131.9$, 132.3, 135.9, 140.2, 148.9, 154.6, 154.9, 162.8 (aromatic carbons), 151.6 (pyrazole C), $169.1(\mathrm{C}=\mathrm{O}) \mathrm{ppm}$. MS $\mathrm{m} / \mathrm{z}$ : found $439\left[\mathrm{M}^{+}\right]$; calcd. 439. Anal. $\mathrm{C}_{26} \mathrm{H}_{21} \mathrm{~N}_{3} \mathrm{O}_{4}$. Found $\mathrm{C} 70.74$ (71.06), H 4.77 (4.82), N 9.42 (9.56).

1-(3-(4-Chlorophenyl)-5-(2-hydroxyphenyl)-4,5-dihydro-1H-pyrazol-1-yl)-2(quinolin-8-yloxy)ethanone $7 \boldsymbol{n}$

Recrystallised from ethanol as brown solid in $93 \%$ yield. m.p. $236-238^{\circ} \mathrm{C}$. IR $(\mathrm{KBr}) v_{\max }$ : 3287 (O-H stretch), 2986 (C-H stretch in aromatics), $1737(\mathrm{C}=\mathrm{O}$ stretch), $1637(\mathrm{C}=\mathrm{N}$ stretch), 1591 ( $\mathrm{C}=\mathrm{C}$ stretch in aromatics), 1178, $1012\left(\mathrm{sp}^{2} / \mathrm{sp}^{3} \mathrm{C}-\mathrm{O}\right.$ stretch$), 1091$ (C-Cl stretch in aromatics) $\mathrm{cm}^{-1}$.

${ }^{1} \mathrm{H}$ NMR $\left(\right.$ DMSO-d $\left._{6}\right): \delta 4.75\left(\mathrm{~s}, 2 \mathrm{H}, \mathrm{OCH}_{2}\right), 3.52-3.57(\mathrm{dd}, \mathrm{J}=4.8,13.2,4.4 \mathrm{~Hz}$, $1 \mathrm{H}$, pyrazole- $\left.\mathrm{H}_{4}\right), 3.67-3.74\left(\mathrm{dd}, \mathrm{J}=12.8,4.8,13.2 \mathrm{~Hz}, 1 \mathrm{H}\right.$, pyrazole- $\left.\mathrm{H}_{4}\right), 5.36(\mathrm{~s}, 1 \mathrm{H}, \mathrm{OH})$, 
5.82-5.86 (dd, J = 4.4, 6.8, $4.8 \mathrm{~Hz}, 1 \mathrm{H}$, pyrazole- $\left.\mathrm{H}_{5}\right), 6.90-7.12(\mathrm{~m}, 4 \mathrm{H}, \mathrm{Ar}-\mathrm{H}), 7.52,7.55$ (d, $\left.\mathrm{J}=8.8 \mathrm{~Hz}, 2 \mathrm{H}, \mathrm{Ar} \mathbf{r}^{\prime} \mathrm{H}\right), 7.96,7.98\left(\mathrm{~d}, \mathrm{~J}=8.8 \mathrm{~Hz}, 2 \mathrm{H}, \mathrm{Ar}{ }^{\prime}-\mathrm{H}\right), 7.50\left(\mathrm{~d}, 1 \mathrm{H}\right.$, quinoline- $\left.\mathrm{H}_{7}\right)$, $7.59\left(\mathrm{t}, 1 \mathrm{H}\right.$, quinoline- $\left.\mathrm{H}_{3}\right), 7.75\left(\mathrm{~d}, 1 \mathrm{H}\right.$, quinoline- $\left.\mathrm{H}_{5}\right), 7.77\left(\mathrm{t}, 1 \mathrm{H}\right.$, quinoline- $\left.\mathrm{H}_{6}\right), 8.25(\mathrm{~d}$, $1 \mathrm{H}$, quinoline- $\left.\mathrm{H}_{4}\right), 8.91\left(\mathrm{~d}, 1 \mathrm{H}\right.$, quinoline- $\left.\mathrm{H}_{2}\right) \mathrm{ppm}$.

${ }^{13} \mathrm{C}$ NMR (DMSO-d $\left.\mathrm{d}_{6}\right): \delta 40.3$ (pyrazole $\left.\mathrm{CH}_{2}\right), 59.8$ (pyrazole $\left.\mathrm{CH}\right), 66.9\left(\mathrm{OCH}_{2}\right), 111.4$, 115.6, 117.9, 121.3, 121.7, 126.4, 126.7, 128.2, 128.3, 129.1, 129.6, 130.8, 134.6, 135.4, 136.5, 140.1, 148.6, 154.8, 155.6 (aromatic carbons), 151.8 (pyrazole $\mathrm{C}$ ), $169.5(\mathrm{C}=\mathrm{O}) \mathrm{ppm}$. MS $m / z$ : found $457\left[\mathrm{M}^{+}\right]$; calcd. 457. Anal. $\mathrm{C}_{26} \mathrm{H}_{20} \mathrm{ClN}_{3} \mathrm{O}_{3}$. Found $\mathrm{C} 67.76(68.20), \mathrm{H} 4.33$ (4.40), N 9.11 (9.18).

\section{1-(5-(2-Hydroxyphenyl)-3-(4-nitrophenyl)-4,5-dihydro-1H-pyrazol-1-yl)-2-} (quinolin-8-yloxy)ethanone 7 o

Recrystallised from ethanol as brown solid in $88 \%$ yield. m.p. $196-198^{\circ} \mathrm{C}$. IR $(\mathrm{KBr}) v_{\max }$ : 3439 (O-H stretch), 3058 (C-H stretch in aromatics), $1662(\mathrm{C}=\mathrm{O}$ stretch), $1603(\mathrm{C}=\mathrm{N}$ stretch), 1528 (N-O stretch), 1418 (C-N stretch, 1090, 1009 ( $\mathrm{sp}^{2} / \mathrm{sp}^{3} \mathrm{C}-\mathrm{O}$ stretch) $\mathrm{cm}^{-1}$.

${ }^{1} \mathrm{H}$ NMR (DMSO-d $\left.{ }_{6}\right): \delta 4.98\left(\mathrm{~s}, 2 \mathrm{H}, \mathrm{OCH}_{2}\right), 3.51-3.56(\mathrm{dd}, \mathrm{J}=12.0,5.6,12.4 \mathrm{~Hz}, 1 \mathrm{H}$, pyrazole- $\left.\mathrm{H}_{4}\right), 3.74-3.79\left(\mathrm{dd}, \mathrm{J}=4.8,12.4,5.2 \mathrm{~Hz}, 1 \mathrm{H}\right.$, pyrazole- $\left.\mathrm{H}_{4}\right), 5.37(\mathrm{~s}, 1 \mathrm{H}, \mathrm{OH}), 5.85-$ $5.88\left(\mathrm{dd}, \mathrm{J}=4.8,6.8,4.4 \mathrm{~Hz}, 1 \mathrm{H}\right.$ pyrazole- $\left.\mathrm{H}_{5}\right), 6.93-7.03(\mathrm{~m}, 4 \mathrm{H}, \mathrm{Ar}-\mathrm{H}), 8.15,8.18(\mathrm{~d}, \mathrm{~J}=$ $9.2 \mathrm{~Hz}, 2 \mathrm{H}, \mathrm{Ar}$ '-H) $, 8.33,8.36\left(\mathrm{~d}, \mathrm{~J}=9.2 \mathrm{~Hz}, 2 \mathrm{H}, \mathrm{Ar} \mathbf{r}^{\prime} \mathrm{H}\right), 7.35\left(\mathrm{~d}, 1 \mathrm{H}\right.$, quinoline- $\left.\mathrm{H}_{7}\right), 7.38$ $\left(\mathrm{t}, 1 \mathrm{H}\right.$, quinoline- $\left.\mathrm{H}_{3}\right), 7.90\left(\mathrm{~d}, 1 \mathrm{H}\right.$, quinoline- $\left.\mathrm{H}_{5}\right), 7.92\left(\mathrm{t}, 1 \mathrm{H}\right.$, quinoline- $\left.\mathrm{H}_{6}\right), 8.38(\mathrm{~d}, 1 \mathrm{H}$, quinoline- $\left.\mathrm{H}_{4}\right), 8.72\left(\mathrm{~d}, 1 \mathrm{H}\right.$, quinoline- $\left.\mathrm{H}_{2}\right) \mathrm{ppm}$.

${ }^{13} \mathrm{C}$ NMR (DMSO-d $): \delta 40.1$ (pyrazole $\left.\mathrm{CH}_{2}\right), 59.8$ (pyrazole $\left.\mathrm{CH}\right), 67.2\left(\mathrm{OCH}_{2}\right), 111.4$, 115.6, 118.3, 121.4, 121.6, 126.7, 126.8, 126.9, 127.8, 128.2, 129.8, 130.4, 135.6, 140.3, 143.4, 149.2, 149.9, 154.6, 155.8 (aromatic carbons), 151.8 (pyrazole C), $169.3(\mathrm{C}=\mathrm{O}) \mathrm{ppm}$. MS $m / z$ : found $468\left[\mathrm{M}^{+}\right]$; calcd. 468. Anal. $\mathrm{C}_{26} \mathrm{H}_{20} \mathrm{~N}_{4} \mathrm{O}_{5}$. Found $\mathrm{C} 66.23$ (66.66), H 4.26 (4.30), N 11.85 (11.96).

\section{Results and Discussion}

A novel series of ring systems derived from quinolin-8-ol 1 have been synthesized in good yields using the synthetic route outlined in Scheme 1 . IR, ${ }^{1} \mathrm{H}$ NMR, ${ }^{13} \mathrm{C}$ NMR, Mass and chemical analysis data were in agreement with the proposed structures of all newly synthesized compounds.

In the IR spectrum of 2, a strong absorption band for carbonyl group at $1730 \mathrm{~cm}^{-1}$ confirms the formation of acetate. ${ }^{1} \mathrm{H}$ NMR spectrum showed a $3 \mathrm{H}$ triplet at $\delta 1.38$ and $2 \mathrm{H}$ quartet at 4.29 corresponding to $\mathrm{CH}_{3}-\mathrm{CH}_{2}$ group and the singlet at 4.95 correspond to $\mathrm{OCH}_{2}$.

In the IR spectrum of $\mathbf{3}$, a broad absorption band at around $3325 \mathrm{~cm}^{-1}$ was due to hydrazide $\mathrm{NH}$ while strong absorption at 1660 was attributed to amide carbonyl. ${ }^{1} \mathrm{H}$ NMR spectrum showed a singlet at $\delta 3.98$ and 9.75 which were accounted for $\mathrm{NH}_{2}$ and $\mathrm{NH}$ and the singlet at 4.87 correspond to $\mathrm{OCH}_{2}$.

In the IR spectrum of $\mathbf{6 a}$, an absorption band at around $1618 \mathrm{~cm}^{-1}$ is attributed to $\mathrm{C}=\mathrm{C}$ stretching and a strong absorption band at $1675 \mathrm{~cm}^{-1}$ corresponds to carbonyl $\mathrm{C}=\mathrm{O}$ stretching. ${ }^{1} \mathrm{H}$ NMR spectrum showed two doublets at 7.54 and 8.06 which were accounted for $\mathrm{CH}=\mathrm{CH}$ and these results confirmed the formation of chalcone.

Reaction of compound $\mathbf{3}$ with $\mathbf{6 a - o}$ in glacial acetic acid resulted in the formation of 1(substituted 3,5-diphenyl-4,5-dihydro-1 $H$-pyrazol-1-yl)-2-(quinolin-8-yloxy)ethanones 7a-o in good yields. IR spectrum of 7 a revealed a band at $1632 \mathrm{~cm}^{-1}$ due to $\mathrm{C}=\mathrm{N}$ stretching in 
pyrazole ring. The ${ }^{1} \mathrm{H}$ NMR spectrum of 7a showed two double doublets at $\delta 3.16-3.21$ and $3.74-3.81$ which indicate the presence of a pair of hydrogens at 4th position and a double doublet at $5.65-5.69$ indicate the presence of one hydrogen at 5 th position in the pyrazole ring. A singlet was observed at $\delta 4.92$ due to $\mathrm{OCH}_{2}$ protons. The mass spectrum of $7 \mathbf{a}$ showed molecular ion peak $\mathrm{M}^{+}$at $\mathrm{m} / \mathrm{z}, 407$ corresponding to molecular formula $\mathrm{C}_{26} \mathrm{H}_{21} \mathrm{~N}_{3} \mathrm{O}_{2}$.<smiles>CCOC(=O)COc1cccc2ccc(-c3ccc4cccc(OCC(N)=O)c4n3)nc12</smiles><smiles>[R]C1=CCCC(O)=C1</smiles>

4<smiles>[R]c1ccc(C(C)=O)cc1</smiles>

5
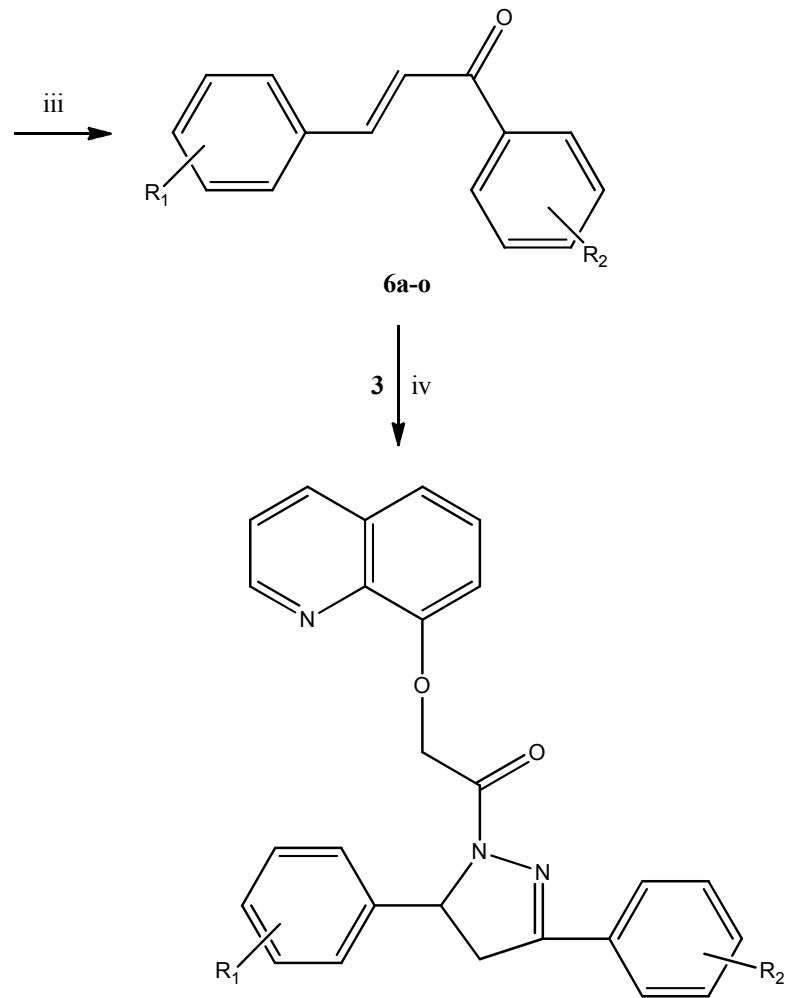

(i) $\mathrm{ClCH}_{2} \mathrm{COOC}_{2} \mathrm{H}_{5}, \mathrm{~K}_{2} \mathrm{CO}_{3}$, DMF (ii) $\mathrm{N}_{2} \mathrm{H}_{4}, \mathrm{H}_{2} \mathrm{O}, \mathrm{ErOH}$ (iii) $\mathrm{NaOH}$, ErOH (iv) Giacial acetic acid $\mathrm{R}_{1}=$ (a) to (e)-H, (f) to (i) $\mathrm{p}-\mathrm{OCH}_{3}$, (k) to (o) o-OH $\mathrm{R}_{2}=$ (a), (f), (k) $-\mathrm{H}$, (b), (g), (l) p-OH, (c), (h), (m) o$\mathrm{OH}$, (d), (i), (n) p-Cl, (e), (i), (o) $\mathrm{p}-\mathrm{NO}_{2}$

Scheme 1. Synthesis of 1-(substituted 3,5-diphenyl-4-5-dihydro-1H-pyrazol-1-yl)-2(quinoline-8-yloxy)ethanones 7a-o 
These newly synthesized quinoline derivatives containing pyrazole 7a-o were screened for their minimum inhibitory concentration by antibacterial activity against two kinds of strains i.e. gram-positive organism Staphylococcus aureus and gram-negative organism Escherichia coli and antifungal activity against Aspergillus niger. The newly synthesized quinoline derivatives containing pyrazole moiety were found potent in the concentration range $100-62.5 \mu \mathrm{g} / \mathrm{mL}$ compared to the standard drugs, $0.19 \mu \mathrm{g} / \mathrm{mL}$ for ofloxacin and $1.56 \mu \mathrm{g} / \mathrm{mL}$ for Fluconazole. The minimum inhibitory concentrations (MICs) of 1-(substituted 3,5-diphenyl-4,5-dihydro-1 $H$-pyrazol-1-yl)-2-(quinolin-8-yloxy)ethanones 7a-o are presented in Table 2.

Table 2. Minimum inhibitory concentration (MICs) of 1-(substituted 3,5-diphenyl-4,5dihydro-1 $H$-pyrazol-1-yl)-2-(quinolin-8-yloxy)ethanones 7a-o

\begin{tabular}{ccccc}
\hline \multicolumn{5}{c}{ Minimum Inhibitory concentration, Concentration in $\mu \mathrm{g} / \mathrm{mL}$} \\
\hline S. No. & Compound & $\begin{array}{c}\text { Gram-positive } \\
\text { organisms }^{\text {a }}\end{array}$ & $\begin{array}{c}\text { Gram-negative } \\
\text { organisms }^{\mathrm{a}}\end{array}$ & Fungi $^{\mathrm{b}}$ \\
\hline 1 & $\mathbf{7 a}$ & 62.5 & 100 & NA \\
2 & $\mathbf{7 b}$ & 75 & 87.5 & NA \\
3 & $\mathbf{7 c}$ & 75 & 87.5 & NA \\
4 & $\mathbf{7 d}$ & 87.5 & 75 & 75 \\
5 & $\mathbf{7 e}$ & 87.5 & 62.5 & 87.5 \\
6 & $\mathbf{7 f}$ & 87.5 & 100 & NA \\
7 & $\mathbf{7 g}$ & 75 & 87.5 & NA \\
8 & $\mathbf{7 h}$ & 75 & 87.5 & NA \\
9 & $\mathbf{7 i}$ & 87.5 & 75 & 87.5 \\
10 & $\mathbf{7 j}$ & 87.5 & 75 & 100 \\
11 & $\mathbf{7 k}$ & 62.5 & 100 & NA \\
12 & $\mathbf{7 l}$ & 75 & 87.5 & NA \\
13 & $\mathbf{7 m}$ & 75 & 87.5 & NA \\
14 & $\mathbf{7 n}$ & 100 & 75 & 75 \\
15 & $\mathbf{7 0}$ & 87.5 & 75 & 75 \\
16 & Ofloxacin & 0.19 & 0.19 & 1.56 \\
17 & Fluconazole & & & . \\
\hline
\end{tabular}

The compounds $\mathbf{7 a}, \mathbf{7 b}, \mathbf{7 c}, \mathbf{7 g}, \mathbf{7 h}, \mathbf{7 k}, \mathbf{7 l}$ and $\mathbf{7 m}$ were more potent against Staphylococcus aureus and $\mathbf{7 d}, \mathbf{7 e}, \mathbf{7 f}, \mathbf{7} \mathbf{i}, \mathbf{7} \mathbf{j}$ and $7 \mathbf{0}$ have moderate potencies. Compound $\mathbf{7 n}$ was weakly potent towards $S$. aureus.

Compound 7e was more potent towards Escherichia coli and $\mathbf{7 d}, \mathbf{7 i}, \mathbf{7 j}, \mathbf{7 n}$ and $7 \mathrm{o}$ have moderate potencies. Compounds $\mathbf{7 a}, \mathbf{7 b}, \mathbf{7 c}, \mathbf{7 f}, \mathbf{7 g}, \mathbf{7 h}, \mathbf{7 k}, \mathbf{7 l}$ and $\mathbf{7 m}$ were weakly potent towards E. coli.

Surprisingly only few compounds showed significant antifungal inhibition with $\mathbf{7 d}, \mathbf{7 n}$ and $7 \mathbf{0}$ being more potent whereas $\mathbf{7 e}, \mathbf{7 i}$ and $\mathbf{7 j}$ were weakly potent towards Aspergillus niger. The results showed that some of the compounds exhibited moderate to good activity against both the strains in antibacterial activity and few compounds were active in antifungal activity.

The studies indicated that variation of substituent in the aromatic rings changes the antibacterial activity significantly. Further compounds containing electron withdrawing groups in Ar' were only potent compared to the other compounds under investigation in antifungal activity. 


\section{Conclusion}

The compounds 1-(substituted 3,5-diphenyl-4,5-dihydro- $1 H$-pyrazol-1-yl)-2-(quinolin-8yloxy)ethanones 7a-o have been successfully synthesized and the structures are established by spectral analysis. The spectral data are consistent with the structure of the newly synthesized compounds. The minimum inhibitory concentration (MIC) of the synthesized compounds was studied using broth dilution method. The results revealed that majority of the tested compounds exhibited moderate to good activity against the control ofloxacin in antibacterial activity and only few compounds exhibit significant antifungal inhibition.

\section{Acknowledgement}

The authors wish to thank the University Grants Commission, New Delhi for award of Junior Research Fellowships to Mr. G. Manjunath, Mr. M. Mahesh and Mr. G. Bheemaraju under SAP and DST, New Delhi for its support under FIST. The authors also thank the Department of Biochemistry, Sri Krishnadevaraya University for helping in anti-microbial screening.

\section{References}

1. Heterocyclic Chemistry by Joule J A, Mills K and Smith G F, $3^{\text {rd }}$ Edition, Reprinted by Stanley Thornes (Publishers) Ltd, UK, 1998.

2. Heterocyclic Chemistry by Thomas L, Gilchrist. $3^{\text {rd }}$ Edition.

3. Sumesh Eswaran, Airody Vasudeva Adhikari, Imran H Chowdhury, Nishith K Pal and Thomas K D, Eur J Med Chem., 2010, 45(8), 3374-3383; DOI:10.1016/j.ejmech.2010.04.022

4. Kumar S, Bawa S and Gupta H, Mini Rev Med Chem., 2009, 9(14), 1648-1654; DOI:10.2174/138955709791012247

5. Akranth. M, Om Prakash T, Rikta Saha, Rahmat Ali, Sandeep Srivastava, Mymoona Akhter, Shaquiquzzaman and Mohammad Mumtaz Alam, Saudi Pharm J., 2013, 21(1), 1-12; DOI:10.1016/j.jsps.2012.03.002

6. Eswaran S, Adhikari A V and Shetty N S, Eur J Med Chem., 2009, 44(11), 46374647; DOI:10.1016/j.ejmech.2009.06.031

7. Nasveld P and Kitchener S, Trans $R$ Soc Trop Med Hyg., 2005, 99(1), 2-5; DOI:10.1016/j.trstmh.2004.01.013

8. Ram Shankar Upadhayaya, Jaya Kishore Vandavasi, Ramakant A Kardile, Santosh V Lahore, Shailesh S Dixit, Hemantkumar S Deokar, Popat D Shinde, Manash P Sarmah and Jyoti Chattopadhyaya, Eur J Med Chem., 2010, 45(5), 1854-1867; DOI:10.1016/j.ejmech.2010.01.024

9. Anna Corradi, Cristina Leonelli, Antonino Rizzuti, Roberto Rosa, Paolo Veronesi, Romano Grandi, Sara Baldassari and Carla Villa, Molecules, 2007, 12(7), 1482-1495; DOI:10.3390/12071482

10. Ranjana Aggarwal, Vinod Kumar, Rajiv Kumar and Shiv P Singh, Beilstein J Org Chem., 2011, 7, 179-197; DOI:10.3762/bjoc.7.25

11. Hany Fakhry Anwara and Mohamed Hilmy Elnagdic, ARKIVOC, 2009, (i), 198-250 (Special Issue Reviews and Accounts).

12. Harish K Arora and Sandeep Jain, Der Pharmacia Lettre, 2013, 5(1), 340-354.

13. Singaravel Mohana, Sarkkarai Ananthan and Kambikudi Raman Murugan, IJPSR, 2010, 1(9), 391-398. 
14. Viral J Faldu, Vrajlal K Gothalia and Viresh H Shah, Indian J Chem., 2015, 54B, 2015, 391-398.

15. Shadab Alam, Rakesh Panda and Monica Kachroo, Indian J Chem., 2014, 53B, 440-443.

16. Tala S D, Vekariya P B, Ghetiya R M, Dodiya B L and Joshi H S, Indian J Chem., 2013, 52B, 807-809.

17. Suvitha Syam, Siddig Ibrahim Abdelwahab, Mohammed Ali Al-Mamary and Syam Mohan, Molecules, 2012, 17(6), 6179-6195; DOI:10.3390/molecules17066179

18. Thanh-Dao Tran, Thi-Thao-Nhu Nguyen, Tuong-Ha Do, Thi-Ngoc-Phuong Huynh, Cat-Dong Tran and Khac-Minh Thai, Molecules, 2012, 17(6), 6684-6696; DOI:10.3390/molecules17066684

19. Jennifer M Andrews, J Antimicrobial Chemotherapy, 2001, 48(S1), 5-16.

20. Determination of minimum inhibitory concentrations (MICs) of antibacterial agents by broth dilution. Clinical Microbiology and Infection, 2003, 9(8) August 2003, 1-7.

21. Mueller J H and Hinton J, Proc Soc Exp Biol Med., 1941, 48, 330-333. 